Pacific Journal of Mathematics

PARACOMPACTIFICATIONS USING FILTER BASES 


\title{
PARACOMPACTIFICATIONS USING FILTER BASES
}

\author{
J. D. WINE
}

\begin{abstract}
During the mid nineteen sixties, C. E. Aull presented a series of papers in which he distinguished several different types of paracompact subsets. Using these concepts, three classes of filter bases are introduced and their convergence and other properties are studied. A variety of characterizations of paracompactness based on the existence of certain types of these filter bases and $z$-filters are given. A paracompactification construction involving the addition of limit points for one of the classes of filter bases is presented in detail. Finally, properties of the paracompactification are explored with some attention given to its ring of continuous functions.
\end{abstract}

Generally, the notation of Gillman and Jerison [7], will be followed. To avoid confusion when considering a property which could be associated with any one of several sets under consideration the set symbol will be affixed before the property symbol.

Filter base classes. We begin with the definitions of the various paracompact subsets which were introduced by Aull.

Definition 1. A subset $M$ of a topological space $X$ is $\alpha$-paracompact if and only if given an $X$-open cover of $M$ there is an $X$-open refinement which covers $M$ and is locally finite at every point $x$ in $X$ (denote by $X$ locally finite).

Definition 2. A subset of $X$ is $\beta$-paracompact if and only if it is paracompact as a subspace of $X$.

Definition 3. A subset $E$ of $X$ is $\sigma$-paracompact if and only if every $X$-open cover of $E$ has an $X$-open $X \sigma$-locally finite refinement which covers $E$.

Definition 4. An $\alpha$-filter base (respectively $\beta$-filter base, $\sigma$-filter base) is a family $\mathscr{F}$ of nonempty zero sets satisfying:

(1) If $Z$ is in $\mathscr{F}$ then $Z$ is not $\alpha$-paracompact (respectively $\beta$-paracompact, $\sigma$-paracompact), and

(2) If $U$ and $V$ are in $\mathscr{F}$ then their intersection contains an element of $\mathscr{F}$.

In this section we wish to develop some properties of the three classes of filter bases defined above. For the most part, the proofs 
are identical for all three types except for the change in terminology. When such is the case we shall give the statement in all three forms, but only prove the result for the $\alpha$-paracompact case, leaving the verifications of the others to the readers.

THEOREM 1. An $\alpha$-filter base (respectively $\beta$-filter base, $\sigma$-filter base) on a locally paracompact space cannot converge. (For properties of a locally paracompact space see [13].)

Proof. By definition a space is locally paracompact if and only if every point has an $\alpha$-paracompact neighborhood. No element of the $\alpha$-filter base can be contained in the $\alpha$-paracompact neighborhood of a point so the $\alpha$-filter base can not converge.

THEOREM 2. If $\mathscr{B}$ is an $\alpha$-filter base (respectively $\beta$-filter base, $\sigma$-filter base), then there is a maximal $\alpha$-filter base (respectively $\beta$-filter base, $\sigma$-filter base) which contains $\mathscr{B}$.

Proof. This is a standard Zorn's lemma argument and is omitted.

Definition 5. Let $X$ be a space, then $\mathscr{A}(X)$ (respectively $\mathscr{B}(X)$, $\mathscr{S}(X)$ ) is the family of all maximal $\alpha$-filter bases (respectively $\beta$-filter bases, $\sigma$-filter bases).

THEOREM 3. If $\mathscr{F}$ is in $\mathscr{A}(X)$ (respectively $\mathscr{B}(X), \mathscr{S}(X)$ ), then any zero set which contains an element of $\mathscr{F}$ is an element of $\mathscr{F}$.

Proof. Let $\mathscr{F}$ be an element of $\mathscr{F}$ which is contained in the given zero set $Z$. Then $Z$ cannot be $\alpha$-paracompact because $F$ is not. For any element $B$ of $\mathscr{F}$, we must have $B \cap Z$ containing $B \cap F$ which contains an element of $\mathscr{F}$. Hence $\{z\} \cup \mathscr{F}$ is an $\alpha$-filter base containing $\mathscr{F}$, which implies $Z$ is an element of $\mathscr{F}$.

THEOREM 4. If $\mathscr{F}$ is in $\mathscr{A}(X)$ (respectively $\mathscr{B}(X), \mathscr{S}(X)$ ), then the intersection of two elements of $\mathscr{F}$ is an element of $\mathscr{F}$.

Proof. The result follows immediately from Theorem 3 and the definition of an $\alpha$-filter base.

As an immediate result of the last two theorems we have the theorem below.

THEOREM 5. If $\mathscr{F}$ is in $\mathscr{A}(X)$ (respectively $\mathscr{B}(X), \mathscr{S}(X)$ ), then $\mathscr{F}$ is a z-filter.

THEOREM 6. An $\alpha$-filter base (respectively $\beta$-filter base, $\sigma$-filter 
base) $\mathscr{F}$ is maximal if and only if every zero set $Z$, such that $Z \cap F$ is not $\alpha$-paracompact (respectively $\beta$-paracompact, $\sigma$-paracompact) for every $F$ in $\mathscr{F}$, is an element of $\mathscr{F}$.

Proof. Necessity. Suppose $\mathscr{F}$ is maximal and let $Z$ be a zero set such that $Z \cap F$ is not $\alpha$-paracompact for every $F$ in $\mathscr{F}$. Then $\mathscr{Q}=\{S: S=F \cap Z, F$ in $\mathscr{F}\} \cup \mathscr{F}$ is a family of non $\alpha$-paracompact zero sets. Now let $S$ and $S^{*}$ be elements of $\mathscr{C}$. If both $S$ and $S^{*}$ are elements of $\mathscr{F}$, then $S \cap S^{*}$ is in $\mathscr{F}$ and hence $\mathscr{U}$. If either one or both of $S$ and $S^{*}$ is not in $\mathscr{F}$, then $S \cap S^{*}$ has the form $F \cap F^{*} \cap Z$ where $F$ and $F^{*}$ are in $\mathscr{F}$. In this case $E=F \cap F^{*}$ is in $\mathscr{F}$, and $E \cap Z$ is an element of $\mathscr{C}$. Therefore, $\mathscr{U}$ is an $\alpha$-filter base, and since $X \cap Z$ is an element of $\mathscr{C}, Z$ is in $\mathscr{C}$. Since $\mathscr{C}$ contains the maximal $\alpha$-filter base $\mathscr{F}$ we must have $\mathscr{Q}=\mathscr{F}$.

Sufficiency. Let $\mathscr{F}$ satisfy the hypothesis, and let $\mathscr{Q}$ be an element of $\mathscr{A}(X)$ which contains $\mathscr{F}$. If $\mathscr{F}$ is not $\mathscr{U}$, then there is a $Z$ in $\mathscr{C}-\mathscr{F}$. Since $\mathscr{C}$ is maximal and contains $\mathscr{F}$, we have $Z \cap F$ is not $\alpha$-paracompact for every $F$ in $\mathscr{F}$. Hence, $Z$ is in $\mathscr{F}$, and $\mathscr{U}$ must equal $\mathscr{F}$.

We shall show later that maximal $\alpha$-filter bases (respectively $\beta$-filter bases, $\sigma$-filter bases) on a Hausdorff completely regular space are very large in the sense that they are almost $z$-ultrafilters, being contained in only one $z$-ultrafilter.

Paracompact spaces and filter bases. We now present a variety of characterizations of paracompactness depending on the existence of $\alpha$-filter bases, $\beta$-filter bases, $\sigma$-filter bases, and $z$-ultrafilters with $\alpha$-paracompact elements. We continue our convention used in the last section regarding the three types of paracompact subsets and the statement and proof of theorems. For some of the results involving $\beta$-filter bases we will have need for the following result.

THEOREM 7. If $\mathscr{F}$ is an $\alpha$-filter base, let $\mathscr{F}^{0}$ be $\left\{F^{0}\right.$ in $\mathscr{F}: F^{0}$ is a neighborhood of some element of $\mathscr{F}\}$. Then $\mathscr{F}$ is a $\beta$-filter base.

Proof. By Theorem 1 in [4], if some element of $\mathscr{F}^{0}$ were $\beta$ paracompact, the element of $\mathscr{F}$ for which it is a neighborhood would be $\alpha$-paracompact.

THeOREM 8. Let $X$ be a completely regular space. Then $X$ is paracompact if and only if there is no free $\alpha$-filter base (respectively: 
$\beta$-filter base, $\sigma$-filter base) on $X$. Also, $X$ is paracompact if and only if there is no free maximal $\alpha$-filter base (respectively $\beta$-filter base, $\sigma$-filter base) on $X$.

Proof. Necessity is obvious for all three cases.

Sufficiency. For the cases involving $\alpha$-filter bases or $\sigma$-filter bases suppose $X$ is not paracompact. Then there is an open cover $\mathscr{G}$ which has no locally finite (respectively $\sigma$-locally finite) refinement. Therefore, if $\mathscr{G}^{*}$ is a finite subfamily of $\mathscr{G}$, the union of elements of $\mathscr{G}^{*}$ is not $X$, and its complement is not $\alpha$-paracompact (respectively $\sigma$-paracompact). Let $\mathscr{B}=\{F: F$ is the complement of the union of a finite subfamily of $\mathscr{G}\}$ and $\mathscr{F}=\{Z$ in $Z(X): Z$ contains some $F$ in $\mathscr{B}\}$. An easy calculation shows $\mathscr{F}$ to be an $\alpha$-filter base (respectively $\sigma$-filter base). Since $\mathscr{G}$ is a cover of $X$, we may use complete regularity to show that $\mathscr{F}$ is free, and we are done.

For the case involving $\beta$-filter bases, we take the $\alpha$-filter base $\mathscr{F}$ obtained above, and use Theorem 7 to obtain the $\beta$-filter base $\mathscr{F}^{0}$. Since $\mathscr{F}$ is free, we may use complete regularity to show that $\mathscr{F}^{0}$ is free.

The last assertion follows from Theorem 2.

COROLlaRY 8A. A completely regular space $X$ is paracompact if and only if every free $z$-filter has an $\alpha$-paracompact (respectively $\beta$-paracompact, $\sigma$-paracompact) element.

Proof. The proof follows from Theorem 5.

CoROLlaRY 8B. Let $X$ be a regular space. Then $X$ is paracompact if and only if there is no $\alpha$-filter base (respectively $\beta$-filter base, $\sigma$-filter base) on $X$. If we drop the condition of regularity then we must drop the case for $\sigma$-filter bases in the conclusion.

Proof. In the proof of the theorem, we needed complete regularity only to obtain $\mathscr{F}$ free, otherwise regularity sufficed, and regularity is needed only for the $\sigma$-filter base.

We note that for Corollary $8 \mathrm{~A}$ results corresponding to Corollary $8 \mathrm{~B}$ can be stated. If the space is locally paracompact and either regular or Hausdorff a slightly nicer result is possible. We will need the following results from [13].

THEOREM 9. A Hausdorff locally paracompact space is regular. 
THEOREM 10. Let $E$ be an $\alpha$-paracompact subset of a regular (respectively Hausdroff) locally paracompact space and let $G$ be any open set containing $E$, then there is a closed $\alpha$-paracompact neighborhood of $E$ contained in $G$.

THEOREM 11. If $X$ is a regular (respectively Hausdorff) locally paracompact space and $A$ is an $\alpha$-paracompact subset, then $A$ is eompletely separated from any disjoint closed set.

Proof. Let $F$ be a closed set disjoint from $A$, then by Theorem 10 there is a closed $\alpha$-paracompact neighborhood $V$ of $A$ which is contained in $X-F$. The subspace $V$ is normal since it is regular and paracompact, hence there is a function $g$ in $C(V)$ such that $0 \leqq g(x) \leqq 1$ and $g$ is zero on $A$ and one on $V$-int $V$. Define $f$ taking $X$ into the closed unit interval by $f \mid V=g$ and $f[X$-int $V]=1$. Clearly $f$ is continuous and completely separates $A$ and $F$.

\section{The Hausdorff case follows from Theorem 9.}

THEOREM 12. If $X$ is a regular (respectively Hausdorff) locally paracompact space and $\mathscr{F}$ is in $\mathscr{A}(x)$, then given any $\alpha$-paracompact set $A$ there is an $F$ in $\mathscr{F}$ which is disjoint from $A$.

Proof. Let $A$ and $\mathscr{F}$ be as in the hypothesis, and let $V$ be a closed $\alpha$-paracompact neighborhood of $A$. For each $F$ in $\mathscr{F}$, the intersection of $F$ and $V$ is $\alpha$-paracompact. Therefore, $E=F \cap(X$-int $V)$ is not $\alpha$-paracompact. Since $E$ is disjoint from $A$, there are disjoint zero set neighborhoods $Z(A)$ and $Z(E)$. By using Theorem 6, it is not difficult to show $Z(E)$ is an element of $\mathscr{F}$.

The Hausdorff case follows from Theorem 9.

COROLlary 12A. If $X$ is a regular (respectively Hausdorff) locally paracompact space, then no maximal $\alpha$-filter base has a cluster point.

Theorem 13. A regular (respectively Hausdorff) locally paracompact space $X$ is paracompact if and only if every free $z$-ultrafilter has an $\alpha$-paracompact element.

Proof. For the nontrivial part, suppose that every free $z$-ultrafilter has an $\alpha$-paracompact element. Then no free $z$-ultrafilter can contain a maximal $\alpha$-filter base by Theorem 12 . Hence, any maximal $\alpha$-filter base on $X$ must be fixed. This is impossible because $X$ is locally paracompact. Hence, $X$ has no maximal $\alpha$-filter base and is paracompact. 
THEOREM 14. If $X$ is a regular (respectively Hausdorff) locally paracompact space, $\mathscr{A}(X)$ and the family of all free $z$-ultrafilters with no $\alpha$-paracompact elements are identical.

Proof. Every such $z$-ultrafilter must be an element of $\mathscr{A}(X)$. Now suppose that $\mathscr{F}$ is in $\mathscr{A}(X)$. Then there is a free $z$-ultrafilter $\mathscr{U}$ containing $\mathscr{F}$ and by Theorem 12 no element of $\mathscr{U}$ is $\alpha$-paracompact. Hence $\mathscr{F}$ equals $\mathscr{U}$.

Paracompactifications using filter bases. In this section we take up the construction of paracompactifications obtained by adding limit points to the various classes of filter bases discussed above.

Definition 6. Let $K$ (respectively $K_{1}, K_{2}$ ) be an index set for the maximal $\alpha$-filter bases (respectively $\beta$-filter bases, $\sigma$-filter bases) on $X$, then define $\mathscr{A}_{F}(X)$ (respectively $\mathscr{B}_{F}(X), \mathscr{S}_{F}(X)$ ) to be $\left\{\mathscr{F}_{k}: k\right.$ is in $K$ (respectively $K_{1}, K_{2}$ ) and $\mathscr{F}_{k}$ is a free maximal $\alpha$-filter base (respectively $\beta$-filter base, $\sigma$-filter base)\}. For each set $G$ in $X$ define $K(G)$ (respectively $K_{1}(G), K_{2}(G)$ ) to be $\left\{k: k\right.$ is in $K$ (respectively $K_{1}, K_{2}$ ) and there is an $F$ in $\mathscr{F}_{k}$ contained in $\left.G\right\}$.

To establish the desired topologies on the extensions of $X$ the following result is needed, its proof is obvious.

THEOREM 15. If $G$ and $H$ are arbitrary sets, then $K(G) \cap K(H)$ equals $K(G \cap H)$ and similarly for $K_{1}$ and $K_{2}$.

Using the previous result, it is a simple computation to obtain the following.

THEOREM 16. Let $\pi X=\left\{y_{k}: k \in K, y_{k}\right.$ is not in $\left.X\right\} \cup X$ and $\mathscr{B}=\left\{H: H=G \cup\left\{y_{k}: k\right.\right.$ is in $\left.K(G)\right\}$ where $G$ is a cozero set $\}$. Then $\mathscr{B}$ is a base for a tapology on $\pi X$. For $K_{1}$ and $K_{2}$ we may define $\pi_{1} X, \mathscr{B}_{1}$, and $\pi_{2} X, \mathscr{B}_{2}$ respectively with the analogous conclusion.

From now on we will be using results from Gillman and Jerison [7], and hence will require all spaces to be Hausdorff and completely regular. Let $W$ (respectively $W_{1}, W_{2}$ ) be the subspace of $\beta X$ obtained by adding to $X$ all points $p$ in $\beta X-X$ for which the $z$-ultrafilter $A^{p}$ on $X$ contains a maximal $\alpha$-filter base (respectively $\beta$-filter base, $\sigma$-filter base). Denote $\left\{A^{p}: p\right.$ is in $W$ (respectively $\left.\left.W_{1}, W_{2}\right)\right\}$ by $A(X)$ (respectively $B(X), S(X)$ ).

Theorem 17. If $\mathscr{F}_{k}$ is in $\mathscr{A}(X)$ (respectively $\mathscr{B}(X), \mathscr{S}(X)$ ) and $A^{p}$ is an element of $A(X)$ (respectively $B(X), S(X)$ ) such that $\mathscr{F}_{k}$ is contained in $A^{p}$, then $\mathscr{F}_{k}$ converges to $p$. 
Proof. Since the zero set neighborhoods of a point in a Hausdorff completely regular space are a base for its neighborhood system, it is sufficient to show that every zero set neighborhood of $p$ contains an element of $\mathscr{F}_{k}$. Let $V(p)$ be a zero set neighborhood of $p$, and let $V^{*}(p)$ be a zero set neighborhood of $p$ contained in the interior of $V(p)$.

Let $E=W$-int $_{W} V(p)$, since $p$ is not in $E$ there are disjoint zero set neighborhoods of $E$ and $p$, call them $F(E)$ and $F(p)$. Let $U(p)$ be the intersection of $F(p)$ and $V^{*}(p)$. Then $U(p)$ is a zero set neighborhood of $p$, and $U(p)$ is contained in int $V_{W} V(p)$. Let $Z(p)=V(p) \cap X$, $Z^{*}(p)=U(p) \cap X$, and $Z(E)=F(E) \cap X$; then $Z(p), Z^{*}(p)$, and $Z(E)$ are zero sets in $X$. We also have $Z(E) \cap Z(p)$ empty and $Z(p) \cup Z(E)=X$. If $p$ is in $X$, then it may be that $Z(p)=V(p)$, or $Z^{*}(p)=U(p)$, but all relations still hold.

Now suppose that $Z(p) \cap F$ is $\alpha$-paracompact for some $F$ in $\mathscr{F}_{k}$. We have that $Z^{*}(p) \cap F$ and $Z(E)$ are disjoint zero sets, and because $Z^{*}(p) \cap F$ must be in $A^{p}, Z(E)$ cannot be in $\mathscr{F}_{k}$. There is an $F^{\prime \prime}$ in $\mathscr{F}_{k}$ such that $F^{\prime \prime} \cap Z(E)$ is $\alpha$-paracompact, but then

$$
F^{\prime} \cap F=\left(\left(F^{\prime \prime} \cap F\right) \cap Z(E)\right) \cup\left(\left(F^{\prime} \cap F\right) \cap Z(p)\right)
$$

which must be $\alpha$-paracompact because components of the union are. This is impossible since $F^{\prime} \cap F$ is in $\mathscr{F}_{k}$. Hence, $Z(E)$ must be in $\mathscr{F}_{k}$ or else $Z(p) \cap F$ is not $\alpha$-paracompact, the former is impossible so the latter is the case. Therefore, $Z(p)$ is in $\mathscr{F}_{k}$.

The case for $\sigma$-filter bases has only the terminology changed in the above proof. The case for $\beta$-filter bases rests upon the fact that in a Hausdorff completely regular space the union of two closed $\beta$-paracompact sets is $\beta$-paracompact, so that the above proof holds with appropriate terminology changes.

COROLlaRY 17A. If $X$ is a Hausdorff completely regular space, there is a one-to-one correspondence between the filter bases in $\mathscr{A}(X)$ (respectively $\mathscr{B}(X), \mathscr{S}(X)$ ) and the z-ultrafilters containing them.

COROLlaRY 17B. Every element in $\mathscr{A}(X)$ (respectively $\mathscr{B}(X)$, $\mathscr{S}(X)$ ) converges in $W$ (respectively $\left.W_{1}, W_{2}\right)$.

If $\mathscr{F}_{k}$ converges to $p$ in $W$, denote $\mathscr{F}_{k}$ by $\mathscr{F}_{k(p)}$. The one-toone correspondence given by Corollary $17 \mathrm{~A}$ illustrates the previously mentioned fact that elements of $\mathscr{A}(X), \mathscr{B}(X)$, and $\mathscr{S}(X)$ are nearly $z$-ultrafilters, since in general, a $z$-filter is contained in many $z$-ultrafilters.

THEOREM 18. Let $U$ be the relative topology of the subspace $W$ of $\beta X$. The family $\mathscr{B}_{w}=\{H: H=G \cup\{p: k(p)$ is in $K(G)\}$ where $G$ is 
a cozero set of $X\}$ is a base for $U$. Analogous statements may be made regarding $W_{1}$ and $W_{2}$.

Proof. Let $Z$ be in $Z(X), G=X-Z$, and $H=W-\operatorname{cl}_{w} Z$. If we show $H$ has the desired form, since $\left\{\operatorname{cl}_{w} Z: Z\right.$ in $\left.Z(X)\right\}$ is a base for the closed sets in $W$, we will have the topology generated by $\mathscr{B}$ contains $\mathscr{K}$.

Let $q$ be an element of $W-X$. Suppose that $q$ is in $H$, then since $\mathscr{F}_{k(q)}$ converges to $q$, and $H$ is a neighborhood of $q$ there is an $F$ in $\mathscr{F}_{k(q)}$ such that $F$ is contained in $H \cap X=G$. Therefore, $\{k(q): q$ is in $H$ \} is contained in $K(G)$. Suppose $q$ is not in $H$. Then $q$ is in $\operatorname{cl}_{w} Z$ and by the construction of $\beta X$ given in [7], $Z$ is an element of $A^{q}$. Hence, since $F$ an element of $\mathscr{F}_{k(q)}$ implies that $F$ is in $A^{q}$, we must have $F \cap Z$ nonempty and no $F$ in $\mathscr{F}_{k(q)}$ is contained in $H \cap X=G$. Therefore, $K(G)$ is contained in $\{k(q): q$ is in $H\}$ and they are equal. Hence, $H=G \cup\{q: q$ is in $H\}=G \cup\{q: k(q)$ is in $K(G)\}$.

To show that the topology generated by $\mathscr{B}$ is contained in $\mathscr{U}$, we show that every set in $\mathscr{B}$ is a member of $\mathscr{C}$. Let $H=G \cup\{p: k(p)$ is in $K(G)\}$ be an element of $\mathscr{B}$. If $p$ is in $H$, then there exists an $F$ in $\mathscr{F}_{k(p)}$ such that $F$ is contained in $G$, so that $F \cap(X-g)$ is empty and the zero set $X-G$ cannot be an element of $A^{p}$. Hence $p$ is not in $\mathrm{cl}_{w}(X-G)$, and $H$ is contained in $W-\mathrm{cl}_{w}(X-G)$. If $p$ is an element of $W-H$, no $F$ in $\mathscr{F}_{k(p)}$ is in $G$. Hence every $F$ in $\mathscr{F}_{k(p)}$ has nonvoid intersection with $X-G$. Therefore, $X-G$ is an element of $A^{p}$ and $p$ is not in $W-\operatorname{cl}_{w}(X-G)$, so that $W-\mathrm{cl}_{w}(X-G)$ is contained in $H$. Hence $H=W-\operatorname{cl}_{w}(X-G)$ and $H$ is an element of $\mathscr{C}$.

Corollary 18A. The space $\pi X$ (respectively $\pi_{1} X, \pi_{2} X$ ) is homeomorphic to a subspace of $\beta X$, namely $W$ (respectively $\left.W_{1}, W_{2}\right)$. Hence $\pi X$ (respectively $\left.\pi_{1} X, \pi_{2} X\right)$ is a completely regular Hausdorff space.

Proof. This follows from Theorems 16, 17, and 18; identifying $\mathscr{B}$ and $\mathscr{B}_{w}$ in the obvious manner.

We now commence a series of lemmas which lead to the result that the extensions $\pi X, \pi_{1} X$, and $\pi_{2} X$ are paracompact. The first two are given simply for reference.

Lemma 19. If $\mathscr{F}$ is a free $z$-filter on a completely regular Hausdorff space, then $\mathscr{F}^{0}$ is also a free $z$-filter.

Lemma 20. Let $E$ be a dense subspace of a completely regular Hausdorff space $Y$. Then, if $\mathscr{F}$ is a free $z$-filter on $Y$ with each 
element having nonvoid interior, the trace of $\mathscr{F}$ on $E$ is a free $z$-filter.

Lemma 21. If a $\pi X$ closed (respectively $\pi_{1} X$ closed, $\pi_{2} X$ closed) set $F$ is contained in $X$, then $F$ is $X$ a-paracompact (respectively $\beta$-paracompact, $X \sigma$-paracompact).

Proof. Suppose that $F$ is not $X \alpha$-paracompact. Then there is an $X$ open cover $\mathscr{G}$ which covers $F$ and has no $X$ locally finite refinement which covers $F$. Hence, $\mathscr{G}^{*}=\mathscr{G} \cup(X-F)$ covers $X$ and has no locally finite refinement which covers $X$. Therefore, $\mathscr{F}=\{Z$ in $Z(X): Z$ contains the complement of the union of a finite subfamily of $\left.\mathscr{G}^{*}\right\}$ is a free $\alpha$-filter base on $X$, and so $F$ is contained in $\mathscr{F}_{k(q)}$, a member of $\mathscr{A}_{F}(X)$. Hence $\{Z$ in $Z(X): F$ is contained in $Z\}$ is contained in $\mathscr{F}_{k(q)}$, which implies $q$ is in $\operatorname{cl}_{\pi X} F=F$, and $F$ is not contained in $X$.

The $\sigma$-paracompact case is analogous with the appropriate changes in terminology.

Now assume $F$ is not $\beta$-paracompact, then $F$ is not $\alpha$-paracompact, and there is a free $\alpha$-filter base $\mathscr{F}$ on $X$ such that $\mathscr{F}$ contains $\{Z$ in $Z(X): Z$ contains $F\}$. For some $z$-ultrafilter $A^{p}$ and some $\mathscr{F}_{k(p)}^{-}$in $\mathscr{A}_{F}(X)$ we have

$$
\mathscr{F}^{0} \subset \mathscr{F} \subset \mathscr{F}_{k(p)} \subset A^{p}
$$

Note that while $A^{p}$ is an element of $A(X)$ we cannot assume $A^{p}$ is in $B(X)$, however this is the case, as we now show. If $Z_{p}$ is the intersection of a zero set neighborhood of $p$ with $X, Z_{p}$ contains an element $Z$ of $\mathscr{F}_{k(p)}$ in $\operatorname{int}_{X} Z_{p}$. Hence $Z_{p}$ is not $\beta$-paracompact. If $F$ is an element of $\mathscr{F}^{0}$, let $Z^{*}$ be an element of $\mathscr{F}$ which is contained in int $_{x} F$, then

$$
F \cap Z_{p} \supset \operatorname{int}_{X}\left(F \cap Z_{p}\right)=\left(\text { int }_{X} F\right) \cap\left(\text { int }_{X} Z_{p}\right) \supset Z^{*} \cap Z
$$

and $F \cap Z_{p}$ is not $\beta$-paracompact. Since the trace $\mathscr{N}$ on the $z$-filter of zero set neighborhoods of $p$ is a $z$-filter on $X$, and since $p$ is a cluster point of $\mathscr{F}^{0}$, the family $\mathscr{N} \cup \mathscr{F}^{0}$ generates a $\beta$-filter base containing $\mathscr{F}^{0}$ and converging to $p$. Hence there is a maximal $\beta$-filter base $\mathscr{B}$ containing $\mathscr{F}^{0}$ and itself contained in $A^{p}$. Therefore $A^{p}$ is in $B(X)$ and $p$ would be in $\mathrm{cl}_{\pi_{1} X} F$, which is a contradiction.

LEMmA 22. Let $f$ be an element of $C(X)$ which is bounded on the complement of an $\alpha$-paracompact (respectively closed $\beta$-paracompact, closed $\sigma$-paracompact) set $A$ by a real number $M$, then there is an extension $f^{\pi}$ (respectively $f^{\prime}, f^{\prime \prime}$ ) of $f$ in $C(\pi X)$ (respectively $C\left(\pi_{1} X\right)$, $C\left(\pi_{2} X\right)$ ) which is bounded on the remainder by $M$. 
Proof. Since any $f$ in $C(X)$ equals $(f \vee 0)+(f \wedge 0)$, it is sufficient to show the result for a function $f \geqq 0$ satisfying the conditions in the hypothesis. Now define $g=(M+1 / 2) \wedge f$, then $g$ is in $C^{*}(X)$, and $g$ restricted to $X-A$ is equal to $f$ restricted to $X-A$. Since $g$ is in $C^{*}(X)$, there is an extension $g^{\pi}$ in $C^{*}(\pi X)$. Define $f^{\pi}$ by $f^{\pi}$ restricted to $X$ is equal to $f$ and $f^{\pi}\left(y_{k}\right)=g^{\pi}\left(y_{k}\right)$.

For each $k, f^{\pi}\left(y_{k}\right) \leqq M$, since if $g^{\pi}\left(y_{k}\right)=r>M$ then the set $S=g^{\pi \leftarrow}[(r-(r-m) / 2, r+(r-m) / 2)]$ is an element of the neighborhood system of $y_{k}$ in $\pi X$. Hence $S$ must contain a non $X \alpha$-paracompact element $Z$ of $\mathscr{F}_{k}$. But $S \cap X$ is contained in $A$ so that $Z$ would be $X \alpha$-paracompact.

Now since $r=g^{\pi}\left(y_{k}\right) \leqq M$, let

$$
V(r)=\left(r-\frac{M+1 / 2-r}{2}, r+\frac{M+1 / 2-r}{2}\right) .
$$

Then $g^{\pi \leftarrow[V(r)]}$ is a neighborhood of $y_{k}$ in $\pi X$. The neighborhood $V(r)$ is contained in the interval $[0, M+1 / 2]$, hence $f^{\pi \leftarrow}[V(r)]=g^{\pi \leftarrow}[V(r)]$. Any neighborhood of $r$ contained in $V(r)$ is the preimage of a neighborhood of $y_{k}$ under $f^{\pi \leftarrow}$. Hence if $U(r)$ is a neighborhood of $r$, then there exists an $\varepsilon$ neighborhood $V_{\varepsilon}(r)$ such that $V_{\varepsilon}(r)$ is contained in $U(r) \cap V(r)$. The set $f^{\pi \leftarrow}\left[V_{\varepsilon}(r)\right]$ is in the neighborhood system of $y_{k}$ in $\pi X$ so that $f^{\pi \leftarrow}[U(r)]$, which contains $f^{\pi \leftarrow}\left[V_{\varepsilon}(r)\right]$, is a neighborhood of $y_{k}$. Therefore, $f^{\pi}$ is continuous at $y_{k}$.

Lemma 23. Let $F$ be an $X$-paracompact (respectively $\sigma$-paracompact) set contained in a $\pi X$ (respectively $\left.\pi_{2} X\right)$ closed neighborhood $V$, which is contained in $X$. Then $F$ is $\pi X \alpha$-paracompact (respectively $\pi_{2} X \sigma$-paracompact).

Proof. Let $\mathscr{G}$ be a $\pi X$ open cover of $F$, and let $\mathscr{G}^{*}=\left\{G^{*}: G^{*}\right.$ is equal to $G \cap \operatorname{int}_{X} V, G$ an element of $\left.\mathscr{G}\right\}$. Since $V$ is $X \alpha$-paracompact by Lemma $21, K\left(\operatorname{int}_{X} V\right)$ is empty. Hence $\operatorname{int}_{X} V=\operatorname{int}_{\pi_{X}} V$ and $\mathscr{G}^{*}$ in both $\pi X$ and $X$ open. We can now obtain an $X$ locally finite is open refinement of $\mathscr{G}^{*}$ which has all of its elements contained in $\operatorname{int}_{\pi X} V$. Hence the refinement of $\mathscr{G}^{*}$ is $\pi X$ open and $\pi X$ locally finite, so $F$ is $\pi X \alpha$-paracompact.

Lemma 24. Let $F$ be an $X \alpha$-paracompact (respectively $\sigma$-paracompact) set with an $X \alpha$-paracompact (respectively $\sigma$-paracompact) neighborhood. Then $F$ is $\pi X \alpha$-paracompact (respectively $\pi_{2} X \sigma$-paracompact).

Proof. Let $G$ be the $X$ open set containing $F$ with $\mathrm{cl}_{X} G$ being $X \alpha$-paracompact. In $\mathrm{cl}_{X} G, F$ and $\mathrm{cl}_{X} G-G$ are closed disjoint sets. 
Since $\operatorname{cl}_{X} G$ is $X \alpha$-paracompact and Hausdorff, it is a normal subspace of $X$. Hence there are disjoint zero set neighborhoods $Z_{1}$ and $Z_{2}$ in $Z\left(\mathrm{cl}_{X} G\right)$ such that $F$ and $\operatorname{cl}_{X} G-G$ are contained in the interior with respect to $\operatorname{cl}_{X} G$ of $Z_{1}$ and $Z_{2}$ respectively. Let $h$ be an element of $C\left(\mathrm{cl}_{X} G\right)$ such that $Z_{1}=h^{\leftarrow}(1), Z_{2}=h^{\leftarrow}(0)$, and $0 \leqq h \leqq 1$.

Define $f$ taking $X$ into the closed unit interval by $f \mid \mathrm{cl}_{X} G=h$ and $f \mid(X-G)=0$. The function $f$ is in $C(X)$. If it is the case that $\mathrm{cl}_{X} G-G$ is empty, we may immediately define $f$ in $C(X)$ by $f[G]=1$ and $f[X-G]=0$.

Let $Z_{3}=\{x: f(x) \geqq 1 / 2\}$. Then we have $F \subset \operatorname{int}_{G} Z_{1} \subset Z_{1} \subset \operatorname{int}_{G} Z_{3} \subset G$. The function $f$ is bounded on the complement of $Z_{3}$ by $1 / 2$, and $Z_{3}$ is $X \alpha$-paracompact. We apply Lemma 22 to get $f^{\pi}$ in $C(\pi X)$ such that $f^{\pi}$ is bounded on the remainder by $1 / 2$. Hence $Z_{1}$ is in $Z(\pi X)$ and is a $\pi X$ closed neighborhood of $F$.

\section{THEOREM 25. $\pi X$ (respectively $\left.\pi_{1} X, \pi_{2} X\right)$ is paracompact.}

Proof. If $\pi X$ is not paracompact, there is a free maximal $\alpha$-filter base $\mathscr{F}$ on $\pi X$ Corollary $8 \mathrm{C}$. By Theorem $5 \mathscr{F}$ is a $z$-filter. Let $\mathscr{F}^{*}$ be the trace of the $z$-filter $\mathscr{F}^{0}$ on $X$. Then $\mathscr{F}^{*}$ is a free $z$-filter.

Suppose $Z^{*}$ is an element of $\mathscr{F}^{*}$, we wish to show that $Z^{*}$ is not $X \alpha$-paracompact. There exists a $Z$ in $\mathscr{F}^{0}$, such that $Z \cap X=Z^{*}$. Let $Z^{\prime}$ be an element of $\mathscr{F}$ such that $Z^{\prime}$ is contained in $\operatorname{int}_{\pi X} Z$. If $Z^{\prime}$ is contained in $X$ then $Z^{\prime}$ is $X \alpha$-paracompact by Lemma 21, and if we assume $Z^{*}$ to be $X \alpha$-paracompact then $Z^{\prime}$ is $\pi X \alpha$-paracompact by Lemma 24. Therefore, $Z^{\prime} \cap(\pi X-X)$ is not empty, but then $\left(\right.$ int $\left._{\pi_{X}} Z\right) \cap(\pi X-X)$ is not empty. For each $y_{k}$ in (int $\left.{ }_{\pi X} Z\right) \cap(\pi X-X)$, the $\operatorname{int}_{\pi X} Z$ is a neighborhood of $y_{k}$. Hence $Z$ must contain an element $F$ of $\mathscr{F}_{k}$. Since $F$ is then contained in $Z^{*}$ also, $Z^{*}$ is not $X \alpha$-paracompact.

Since $\mathscr{F}^{*}$ is an $\alpha$-filter base on $X$, there is a maximal $\alpha$-filter base $\mathscr{N}^{*}$ on $X$ which contains $\mathscr{F}^{*}$, and since $\mathscr{F}^{*}$ is free so is $\mathscr{N}^{*}$. Hence $\mathscr{N}^{*}$ converges to some $y_{k}$ in $\pi X-X$. Let $\mathscr{U}^{*}$ be the unique $z$-ultrafilter on $X$ containing $\mathscr{N}^{*}$. Let $\overline{\mathscr{Q}}$ be the $z$-filter on $\pi X$ generated by $\left\{\mathrm{cl}_{\pi X} Z: Z\right.$ is in $\left.\mathscr{Q}^{*}\right\}$. The set of cluster points of $\overline{\mathscr{U}}$ contains $y_{k}$, so there is a $z$-ultrafilter $\mathscr{U}$ on $\pi X$ containing $\overline{\mathscr{U}}$ and converging to $y_{k}$.

Since $\mathscr{F}$ is a free $z$-filter on $\pi X$, it cannot be contained in the convergent $z$-ultrafilter $\mathscr{U}$. Therefore, there is an $E$ in $\mathscr{F}$ and an $E^{*}$ in $\mathscr{C}$ which are disjoint. Hence $E$ and $E^{*}$ have disjoint zero set neighborhoods $V$ and $V^{*}$. The zero set $V$ is in $\mathscr{F}^{0}$ which implies $V \cap X$ is in $\mathscr{F}^{*}$ and $V$ contains $\operatorname{cl}_{\pi X}(V \cap X)$ so $V$ is in $\overline{\mathscr{C}}$. Therefore, $V$ and $V^{*}$ are both elements of $\mathscr{U}$, which is absurd. We have a contradiction and the proof for the space $\pi X$ is complete. 
The proof for the space $\pi_{2} X$ requires only the appropriate terminology changes in the above. For the space $\pi_{1} X$, we may use the fact that $\beta$-paracompactness is a property which is dependent only on the set, so the application of Lemma 24 is not necessary. Other than this change the proof is analogous to that for $\pi X$.

COROLLARY 25A. The three paracompactifications are $T_{4}$ spaces.

The question as to the relation which exist among $\pi X, \pi_{1} X$, and $\pi_{2} X$ is answered in the following.

THEOREM 26. The three paracompactifications constructed above are identical.

Proof. Since all the paracompactifications are subsets of $\beta X$, we need only show that for points in the remainder, the families of $z$-ultrafilters are identical.

Let $A^{p}$ be a $z$-ultrafilter converging in $\pi X-X$ with its associated maximal $\alpha$-filter base $\mathscr{F}_{k(p)}$. Let $V$ be a basic open set in $\pi X$ containing $p$. Then there is an element $F$ of $\mathscr{F}_{k(p)}$ contained in $V \cap X$. The set $Z=X-(V \cap X)$ is a zero set of $X$. Since $Z$ and $F$ are disjoint zero sets, they have disjoint zero set neighborhoods $Z^{0}$ and $F^{0}$. The set $F^{0}$ is an element of the $\beta$-filter base $\mathscr{F}_{k(p)}^{0}$, and $F^{0}$ is contained in $V \cap X$. Hence $\mathscr{F}_{k(p)}^{0}$ converges to $p$. We can then find a free maximal $\beta$-filter base converging to $p$ and contained in $A^{p}$. Therefore, $A^{p}$ is in $B(X)$.

Now suppose $A^{p}$ is a $z$-ultrafilter on $X$ converging to a point in $\pi_{1} X-X$ with its associated maximal $\beta$-filter base $\mathscr{F}_{k(p)}$. Now $\mathscr{F}_{k(p)}$ is a free $\alpha$-filter base which has a unique $z$-ultrafilter $A^{p}$ containing it. Hence the free maximal $\alpha$-filter base containing $\mathscr{F}_{k(p)}$ must be contained in $A^{p}$, and $A^{p}$ is in $A(X)$. We have $A(X)=B(X)$ and $\pi X$ is identical to $\pi_{1} X$.

A similar argument established $\pi_{1} X$ to be identical to $\pi_{2} X$.

THEOREM 27. A completely regular Hausdorff space $X$ is locally paracompact if and only if $X$ is open in $\pi X$.

Proof. The proof of this is a straight forward argument and so is omitted.

THEOREM 28. If $X$ is a Hausdorff locally paracompact space, then $F$ is $X$ Q-paracompact if and only if $\mathrm{cl}_{\pi X} F=F$.

Proof. Necessity. If $F$ is $X \alpha$-paracompact, then for each $y_{k}$ in 
$\pi X-X$ we may apply Theorem 11 to get a zero set $Z_{k}$ in $\mathscr{F}_{k}^{0}$ which is disjoint from $F$. The set given by $\left(\right.$ int $\left._{X} Z_{k}\right) \cup\left\{Y_{j}: j\right.$ is in $K\left(\right.$ int $\left.\left._{X} Z_{k}\right)\right\}$ is a neighborhood of $y_{k}$ in $\pi X$ and is disjoint from $F$.

Sufficiency. This follows from Lemma 21.

CoROLlARY 28A. If $X$ is a locally paracompact Hausdorff space, and $F$ is an $X$-paracompact set, then $F$ is $\pi X \alpha$-paracompact.

THEOREM 29. Let $X$ be Hausdorff and completely regular, let $G$ be an open subset of $X$, and let $G^{*}=G \cup\left\{y_{k}: k\right.$ is in $\left.K(G)\right\}$. Then $\mathrm{cl}_{\pi X} G=\mathrm{cl}_{\pi X} G^{*}$.

Proof. Clearly $G$ is contained in $G^{*}$, so that $\mathrm{cl}_{\pi X} G$ is contained in $\mathrm{cl}_{\pi X} G^{*}$. If $y_{k}$ is in $G^{*}$, then there is an $F_{k}$ in $\mathscr{F}_{k}$ such that $F_{k}$ is contained in $G$. Since $\mathscr{F}_{k}$ converges to $y_{k}$, every neighborhood of $y_{k}$ has nonvoid intersection with $F_{k}$ and hence $G$. Therefore, $G^{*}$ is contained in $\mathrm{cl}_{\pi X} G$, and so $\mathrm{cl}_{\pi X} G^{*}$ equals $\mathrm{cl}_{\pi X} G$.

THEOREM 30. Let $\nu X$ be the Hewitt Realcompactification of $X$. Then $\nu X$ is contained in $\pi X$. (This result and the next require all cardinals to be nonmeasurable.)

Proof. We know $\nu X$ is the smallest realcompactification of $X$. Since $\pi X$ is paracompact, it is realcompact and $\nu X \cap \pi X$ is realcompact. Hence $\nu X$ must be contained in this intersection.

THEOREM 31. For a Hausdorff completely regular space $X$ the following are equivalent.

(1) $\pi X$ is identical to $\nu X$.

(2) $A(X)$ is identical to the family of real z-ultrafilters on $X$.

(3) For each $f$ in $C(X)$ and each $\mathscr{F}_{k}$ in $\mathscr{A}_{F}(X)$ there is an element $Z_{f}$ of $\mathscr{F}_{k}$ such that $f$ is bounded on $Z_{f}$.

Proof.

$(1) \Rightarrow(2)$ This is obvious.

(2) $\Rightarrow(3)$ If $A(X)$ is identical with the family of real $z$-ultrafilters on $X$, then every $\mathscr{F}_{k}$ in $\mathscr{A}_{F}(X)$ is contained in a unique $z$-ultrafilter with c.i.p. If some $f$ in $C(X)$ is unbounded on every element of $\mathscr{F}_{k}$, then for every positive integer $n, Z_{n}=\{x:|f(x)| \geqq n\}$ has nonvoid intersection with every $Z$ in $\mathscr{F}_{k}$. Hence $\mathscr{F}_{k} \cup\left\{Z_{n}: n=1\right.$, $2, \cdots\}$ generates a $z$-filter which contains $\mathscr{F}_{k}$ and does not have c.i.p. This is a contradiction.

$(3) \Rightarrow(1)$ Let $\mathscr{F}_{k(q)}$ be an element of $\mathscr{A}_{F}(X)$. We show that 
$A^{q}$ is real. Let $f^{*}$ be the Stone extension of $f$ into the one-pointcompactification of the real numbers (see $\S 7.5$ of [7]). Then by Theorems 5.7 and 7.6 in [7] we have $f^{*}(q) \neq \infty$, since $Z_{f}$ is an element of $A^{q}$ for each $f$ in $C(X)$. Hence $A^{q}$ is real, $\pi X$ is contained in $\nu X$.

We shall continue to use $f^{*}$ to represent the Stone extension of a function $f$ in $C(X)$ into the one-point-compactification of the real numbers.

THEOREM 32. Let $X$ be a Hausdorff completely regular space, and let $f$ be in $C(X)$. Then there is a continuous extension $f^{\pi}$ in $C(\pi X)$ of $f$ if and only if the z-filter $f^{\sharp}\left(\mathscr{F}_{k}\right)=\{Z$, a zero set in the real numbers: $f^{\leftarrow}[Z]$ is in $\left.\mathscr{F}_{k}\right\}$ converges, for every $\mathscr{F}_{k}$ in $\mathscr{A}_{F}(X)$. (For properties of $f^{\sharp}$ see $\S \S 4.12$ and 10.17 of [7].)

Proof. Necessity. Since $f^{\pi}$ is continuous, the filter generated by the image of the neighborhood system of $y_{k}$ in $\pi X$ converges. Now $\mathscr{F}_{k}$ is a base for a filter $\mathscr{F}$ which contains the neighborhood system of $y_{k}$ in $\pi X$. Therefore the filter generated by $\left\{S: S=f^{\pi}[F]\right.$ where $F$ is in $\left.\mathscr{F}_{k}\right\}$ converges to the real number $r=f^{\pi}\left(y_{k}\right)$. Hence every zero set neighborhood of $r$ is an element of this filter. Since every $F$ in $\mathscr{F}_{k}$ is contained in $X$, the filter generated by $\left\{S: S=f^{\pi}[F], F\right.$ is in $\left.\mathscr{F}_{k}\right\}$ equals the filter generated by $\left\{S: S=f[F], F\right.$ is in $\left.\mathscr{F}_{k}\right\}$. For each zero set neighborhood $V(r)$ of $r, f^{\pi \leftarrow}[V(r)]$ is a zero set neighborhood of $y_{k}$, so $f^{\pi \leftarrow}[V(r)] \cap X$ is in $\mathscr{F}_{k}$, and equals $f^{\leftarrow}[V(r)]$ giving $f^{\#}\left(\mathscr{F}_{k}^{-}\right)$convergent.

Sufficiency. To show $f$ has an extension to $\pi X$ it is sufficient to show that the limit of the filter generated by $S=\{f[X \cap V]: V$ is a neighborhood of $y_{k}$ in $\pi X$ \} exists for every $k$ in $K$. Let $r$ be the limit of $f^{\sharp}\left(\mathscr{F}_{k}\right)$. Then if $V(r)$ is any zero set neighborhood of $r$, there is a $Z$ in $f^{\sharp}\left(\mathscr{F}_{k}\right)$ which is contained in int $V(r)$. Hence $f^{\leftarrow}[Z]$ is in $\mathscr{F}_{k}$ so that $f^{\leftarrow}[V(r)]$ contains an element of $\mathscr{F}_{k}$, and $f\left(f^{\leftarrow}[V(r)]\right)=V(r)$ is an element of $S$, and we are through.

Definition 7. For a Hausdorff completely regular space $X$, define $C^{\approx}(X)=\left\{f\right.$ in $C(X): f^{*}(p) \neq \infty$ for every $p$ in $\left.\pi X\right\}$.

We note without proof that $C^{\pi}$ is identical to $\{f$ in $C(X): \pi X$ is contained in $\left.\nu_{f} X\right\}$ where $\nu_{f} X$ is the realcompact subspace of $\beta X$ equal to $\left\{p\right.$ in $\left.\beta X: f^{*}(p) \neq \infty\right\}$. Hence $\pi X=\cap\left\{\nu_{f} X: f\right.$ is in $\left.C^{\pi}\right\}$. (See 8B2 and $8 \mathrm{~B} 3$ in [7].)

Theorem 33. Let $f$ be in $C(X)$. Then $f$ has an extension $f^{\pi}$ in $C(\pi X)$ if and only if $f$ is in $C^{\pi}(X)$. 
Proof. Necessity. If $f$ has an extension $f^{\pi}$ in $C(\pi X)$, then $f^{*}$ and $f^{\pi}$ must agree on the dense subset $X$ of $\pi X$. Both $f^{\pi}$ and $f^{*}$ may be considered as functions into the one-point-compactification of the real numbers. Hence both are defined for all of $\pi X$ and must agree. Since $f^{\pi}(p) \neq \infty$ for all $p$ in $\pi X$, neither is $f^{*}$, and $f$ is in $C^{\pi}(X)$.

Sufficiency. If $f$ is in $C^{\pi}(X), f^{*}$ is a real valued continuous extension of $f$ to all of $\pi X$.

Corollary 33A. The family $C^{\pi}(X)$ is a subring of $C(X)$ and is isomorphic to $C(\pi X)$.

Theorem 34. Let $f$ be an element of $C(X)$. Then $Z_{f}=\{p$ in $\left.\beta X: f^{*}(p) \neq \infty\right\}$ is a zero set in $\beta X$.

Proof. Define $g=|f| \vee 1$. Then $Z_{f}=Z_{g}$ and it will suffice to show the result for functions bounded away from zero and positive. Assume $f \geqq 1$ and define $h=1 / f$ then $h$ is in $C^{*}(X)$ and has an extension $h^{\beta}$ in $C(\beta X)$. The functions $h^{\beta}$ and $h^{*}$ are equal. Now $f^{*} \mid\left(\beta X-Z\left(h^{\beta}\right)\right)$ and $1 / h^{\beta} \mid\left(\beta X-Z\left(h^{\beta}\right)\right)$ are both extensions of $f$ and must be equal. Hence $Z_{f}$ and $Z\left(h^{\beta}\right)$ are equal, for otherwise $h^{\beta} f^{*}$ would equal 1 for some point in $Z_{f}$ or $Z\left(h^{\beta}\right)$.

TheOREM 35. If $\beta X-\pi X$ is not empty, it has carinality greater than or equal to $2^{c}$.

Proof. Let $f$ be in $C^{\pi}(X)$ and let $Z_{f}$ be nonvoid. Then $Z_{f}$ is contained in $\beta X-\pi X$. Since $Z_{f}$ intersects the closure of $\pi X$ but not $\pi X \cup X$ we apply Theorem 9.4 in [7] to get the result.

DeFinition 8. A function $f$ in $C(X)$ bounded on the complement of an $X \alpha$-paracompact set is essentially bounded.

TheOREM 36. A function $f$ in $C(X)$ has an extension $f^{\pi}$ in $C(\pi X)$ bounded on the remainder if and only if it is essentially bounded.

Proof. Necessity. If $f$ in $C(X)$ has the extension $f^{\pi}$ in $C(\pi X)$ with bound $M$ on the remainder, then for each $y_{k}$ in the remainder the set $V_{k}(\varepsilon)=f^{\pi \leftarrow}\left[\left(f\left(y_{k}\right)-\varepsilon / 2, f\left(y_{k}\right)+\varepsilon / 2\right)\right]$ is an open neighborhood of $y_{k}$ for $\varepsilon>0$. The set $\pi X-\cup\left\{V_{k}(\varepsilon): y_{k}\right.$ is in $\left.\pi X-X\right\}$ is an $X$ $\alpha$-paracompact set which has $f$ bounded by $M+\varepsilon / 2$ on its complement.

Sufficiency. This is simply Lemma 22.

TheOREM 37. If $\pi X-X$ is pseudocompact, then the family of 
essentially bounded functions on $X$ is $C^{\pi}(X)$.

We wish to give some results now which present properties of $\alpha$-filter bases in relation to various other properties.

THEOREM 38. The maximal $\alpha$-filter bases are prime $z$-filters.

Proof. This is an easy computation and is omitted.

We recall that $\mathscr{F}_{k(q)}$ is the unique free $\alpha$-filter base on $X$ contained in the $z$-ultrafilter $A^{q}$.

THEOREM 39. If $\mathscr{F}_{k}$ is in $\mathscr{A}(X)$, then $\mathscr{F}_{k(q)}$ contains $Z\left[0^{q}\right]$ (for properties of $0^{q}$ see $4 \mathrm{I}, 7.12-7.15,7 \mathrm{H}$ in [7]).

Proof. Every prime ideal in $C(X)$ is contained in a maximal ideal $M^{p}$, and contains an $0^{p}$ for unique $p . Z^{-}\left[\mathscr{F}_{k}^{-}\right]$is a prime ideal. Hence $Z\left[Z^{\leftarrow}\left[\mathscr{F}_{k(q)}\right]\right]$ contains $Z\left[0^{q}\right]$.

CoRollary 39A. If $X$ is a P-space, then $\mathscr{F}_{k(p)}=Z\left[0^{p}\right]$ (for properties of $P$-spaces see $4 \mathrm{~J}, \mathrm{~K}, \mathrm{~L}, 5 \mathrm{P}$, and $7 \mathrm{~L}$ in [7]).

Corollary 39B. If $X$ is a P-space, then $c l_{n X} Z=Z$ for $\alpha$-paracompact $Z$.

THEOREM 40. Let $\mathscr{F}_{k}$ be a free $\alpha$-filter base such that the open cover consisting of the complements of members of $\mathscr{F}_{k}$ has no locally finite open refinement which covers $X$. Then the intersection of any subfamily $\mathscr{F}^{*}$ of $\mathscr{F}_{k}$, which has locally finite complements, is a member of $\mathscr{F}_{k}$.

Proof. Let $\mathscr{F}_{k}$ be an element of $\mathscr{A}_{F}(X)$, and let $\mathscr{G}$ be $\{G: G=$ $X-Z, Z$ in $\left.\mathscr{F}_{k}^{-}\right\}$. Since $\mathscr{G}$ has no locally finite refinement which covers $X$, every locally finite subfamily $\mathscr{G}^{*}$ of $\mathscr{G}$ must have $\bigcap\left\{Z: Z=X-G, G\right.$ in $\left.\mathscr{G}^{*}\right\}$ to be nonvoid. Let $Z^{*}$ be such an intersection. We show $Z^{*}$ to be in $F_{k}$. If $Z^{*}$ is not in $F_{k}$, then there is an $F$ in $\mathscr{F}_{k}$ such that $F \cap Z^{*}$ is $\alpha$-paracompact. Then $\mathscr{P}=\{G: G$ is in $\mathscr{G}^{*}$ or $\left.G=X-F\right\}$ is an open locally finite refinement of $\mathscr{G}$. There is an open locally finite refinement $\mathscr{L}^{*}$ of $\mathscr{G}$ which covers $F \cap Z^{*}$. Then $\mathscr{H} \cup \mathscr{C}^{*}$ is a locally finite open refinement of $\mathscr{G}$ which covers $X$, and we have reached a contradiction.

THeOREM 41. Let $X$ be a space such that for every $\mathscr{F}_{k}$ in $\mathscr{A}_{k}(X)$, the family $\left\{G: G=X-Z, Z\right.$ in $\left.\mathscr{F}_{k}\right\}$ has no open locally finite refinement which covers $X$. Then for any paracompactification $Y$ of $X$ every 
$\mathscr{F}_{k}$ in $\mathscr{A}(X)$ must converge in $Y$.

Proof. Suppose that $\mathscr{F}_{k}$ in $\mathscr{A}_{F}(X)$ does not converge in $Y$. Let $\mathscr{F}=\left\{F: F=\mathrm{cl}_{y} \mathrm{Z}, Z\right.$ in $\left.\mathscr{F}_{k}\right\}$. Then since $\mathscr{F}_{k}$ does not converge in $Y$, $\{G: G=y-F, F$ in $\mathscr{F}\}$ is a $Y$ open cover of $Y$. Since $Y$ is paracompact, there is a $Y$ open locally finite refinement $\mathscr{G}^{*}=\left\{G_{j}^{*}: j\right.$ is in $J\}$ which covers $Y$. Then $\left\{G_{j}: G_{j}=G_{j}^{*} \cap X, G_{j}^{*}\right.$ in $\left.\mathscr{G}^{*}\right\}$ is an $X$ open locally finite refinement of $\left\{G: G=X-Z, Z\right.$ in $\left.\mathscr{F}_{k}\right\}$ which covers $X$.

THEOREM 42. Let $X$ be a space such that for every $\mathscr{F}_{k}$ in $\mathscr{A}_{F}(X)$, the family $\left\{G: G=X-Z, Z\right.$ in $\left.\mathscr{F}_{k}\right\}$ has no open locally finite refinement which covers $X$. Then if $Y$ is any Hausdorff paracompactification of $X$, there exists a continuous function $f: \pi X \rightarrow Y$ that holds $X$ pointwise fixed.

Proof. Define the function $g: X \rightarrow Y$ by $g(x)=x$. Let $\bar{g}$ be the Stone extension of $g$ taking $\beta X$ into $\beta Y$ and define $X_{0}=\bar{g}^{-}[Y]$. Let $\bar{f}=\bar{g} \mid X_{0}$. Then $\bar{f}$ is continuous, onto $Y$, and holds $X$ fixed. Since $\mathscr{F}_{k}$ in $\mathscr{A}_{F}(X)$ must converge in $Y$ to some point $y, \mathscr{F}_{k}$ must converge to some element in $\bar{f}^{\leftarrow}(y)$. Therefore, $\pi X$ is contained in $X_{0}$, and $f=\bar{f} \mid \pi X$ is the desired function.

CoRollary 42A. If $X$ is a space such that for every $\mathscr{F}_{k}$ in $\mathscr{A}_{F}(X)$, the family $\left\{G: G=X-Z, Z\right.$ in $\left.\mathscr{F}_{k}\right\}$ has no locally finite open refinement which covers $X$, then $\pi X$ is the smallest paracompactification contained in $\beta X$.

THEOREM 43. Let $X$ be dense in a Hausdorff completely regular extension $Y$ such that all $\mathscr{F}_{k}^{-}$in $\mathscr{A}_{F}(X)$ converge in $Y$. Then $Y$ contains a paracompactification of $X$. Further, if $Y-X$ consists only of limit points of the $\mathscr{F}_{k}$ in $\mathscr{A}_{F}(X)$ then $Y$ is paracompact.

Proof. Let $f: X \rightarrow Y$ be the identity map, let $\bar{f}$ be the Stone extension of $f$ into $\beta Y$, and let $X_{0}=\bar{f}^{-}[Y]$. If $f_{0}=\bar{f} \mid X_{0}$, then $f_{0}$ is a closed continuous extension of $f$ onto $Y$, and $f\left[X_{0}-X\right]$ is contained in $Y-X$. (See $\S \S 10.13$ and 10.15 in [7].)

We show that $X_{0}$ contains $\pi X$. Let $p$ be an element in $\pi X-X$, let $y_{p}$ be an element of $Y$ such that $A^{p}$ converges to $y_{p}$ in $Y$, and let $y=f(p)$. Suppose that $y$ is not identical to $y_{p}$. Then there are disjoint open neighborhoods $V(y)$ and $V\left(y_{p}\right)$ in $Y$. If $V(p)=\bar{f} \leftarrow[V(y)]$, $G=\bar{f}^{-}\left[V\left(y_{p}\right)\right]$, then we must have $V(p) \cap G$ empty. Since $A^{p}$ converges to $y_{p}$ there is a $Z_{p}$ in $A^{p}$ that is contained in $V\left(y_{p}\right)$, and hence $G$, since $f$ is the identity. But $V(p)$ is a neighborhood of $p$ in $\pi X$, so that there is an element $Z_{y}$ of $A^{p}$ contained in $V(p)$. Hence $Z_{y}$ does not 
intersect $Z_{p}$ which is impossible so $y_{p}=y$, and $\pi X$ is contained in $X_{0}$. The set $f_{0}[\pi X]$ contains $X$, and is $\beta$-paracompact in $Y$, since $f_{0}$ is a closed continuous function. Therefore, $f_{0}[\pi X]$ is a paracompactification of $X$ contained in $Y$.

The second assertion follows, since if $Y$ satisfies the given condition, $f_{0}[\pi X]=Y$, and $X_{0}=\pi X$.

THeORem 44. Let $X$ be a space such that for every $\mathscr{F}_{k}$ in $\mathscr{A}_{F}(X)$, the family $\left\{G: G=X-Z, Z\right.$ in $\left.\mathscr{F}_{k}\right\}$ has no open locally finite refinement which covers $X$, and let $X$ be dense in a Hausdorff completely regular extension $Y$. Then $Y$ contains a paracompactification of $X$ if and only if $Y-X$ contains a limit point for every $\mathscr{F}_{k}$ in $\mathscr{A}_{F}(X)$.

Proof. The proof follows from Theorems 41 and 43 .

THEOREM 45. If $Y$ is a Hausdorff paracompactification of $X$ such that some $\mathscr{F}_{k}$ in $\mathscr{A}_{F}(X)$ does not converge in $Y$, then there is an $X$ locally finite partition of unity $\mathscr{L}$ contained in $Z_{x}^{\leftarrow}\left[\mathscr{F}_{k}\right]$.

Proof. Suppose that $\mathscr{F}_{k}$ is in $\mathscr{A}_{F}(X)$ and does not converge in $Y$. Then $\left\{\operatorname{cl}_{Y} Z: Z\right.$ is in $\left.\mathscr{F}_{k}\right\}$ has void intersection and generates the free $z$-filter $\mathscr{F}^{*}$ on $Y$. Let $\mathscr{G}=\left\{G: G=Y-Z, Z\right.$ in $\left.\mathscr{F}^{*}\right\}$. Then $\mathscr{G}$ is an open cover of $Y$. Since $Y$ is Hausdorff, there is a locally finite partition of unity subordinate to $\mathscr{G}$, call it $\mathscr{E}$. If $f$ is in $\mathscr{F}$, then $Z_{Y}(f)$ contains some $Z$ from $\mathscr{F}^{*}$ Hence $f \mid X$ is in $Z_{X}^{+}\left[\mathscr{F}_{k}\right]$, and there is the subfamily $\mathscr{L}=\{f \mid X: f$ is in $\mathscr{E}\}$ of $Z_{X}^{\leftarrow}\left[\mathscr{F}_{k}\right]$ which is an $X$ locally finite partition of unity.

THeOREM 46. Each $\mathscr{F}_{k}$ in $\mathscr{A}_{F}(X)$ has the property that the family $\left\{G: G=X-Z, Z\right.$ is in $\left.\mathscr{F}_{k}\right\}$ has no locally finite open refinement which covers $X$ only if $Z^{\leftarrow}\left[\mathscr{F}_{k}\right]$ contains no locally finite partition of unity.

Proof. A locally finite partition of unity which is contained in $Z^{-}\left[\mathscr{F}_{k}\right]$ yields a locally finite open refinement of $\{G: G=X-Z, Z$ in $\left.\mathscr{F}_{k}\right\}$ by cozero sets.

THEOREM 47. A space $X$ is nonparacompact if and only if for each free $z$-filter $\mathscr{F}$ there is a family $\mathscr{G}^{*}$ which consists of complements of elements of $\mathscr{F}$ and has no locally finite open refinement.

Proof. Necessity. Let $\mathscr{F}$ be a free $z$-filter, and let $\mathscr{G}=\left\{G_{j}: j\right.$ is in $J\}$ be an open cover of $X$ which has no open locally finite refinement. For each $x$ in $X$, let $G_{x}$ be an element of $\mathscr{G}$ which contains $x$. Let $Z$ be a zero set which contains $x$ in its complement, and such 
that the complement of $Z$ is contained in $G_{x}$. For each $x$ in $X$ let $Z_{x}$ be that element of $\mathscr{F}$ which contains $x$ in its complement. Then the family $\mathscr{G}^{*}=\left\{X-F_{x}: F_{x}=Z \cup Z_{x}, x\right.$ in $\left.X\right\}$ is the desired family of open sets.

\section{Sufficiency. This is immediate.}

J. Van der Slot [12] and H. Herrlich ([8], [9], [10]) have done work pertaining to extensions of spaces. If $\beta$ is a property of topological spaces a $\beta$-extension of $X$ is a space $\gamma X$ containing a dense homeomorphic image of $X$ and having property $\beta$. A $\beta$-extension $\gamma X$ of $X$ is maximal if for each continuous function $f$ of $X$ into a space $Y$ having property $\beta$ there is a continuous extension of $f$ to all of $\gamma X$. A space $X$ is $\beta$-regular if it is homeomorphic to a subspace of a space which is the product of spaces each having property $\beta$. The following theorem is due to Van der Slot [12].

THEOREM 48. Let $\beta$ be a property possessed by Hausdorff spaces. Then a $\beta$-regular Hausdorff space has a maximal $\beta$-extension if and only if $\beta$ is closed hereditary and productive.

From the above theorem and the fact that paracompactness is not productive we have the following:

THEOREM 49. A Hausdorff space $X$ does not in general possess a maximal paracompactification.

It would be of interest to obtain a characterization of those spaces $X$ which have $A_{F}(X)$ such that if $F$ is in $A_{F}(X)$ then $\{G: G=X-Z$, $Z$ in $F$ \} has no locally finite open refinements. It would also be interesting to know if it is true in general that $\pi X$ is the smallest paracompactification of $X$ contained in $\beta X$.

\section{REFERENCES}

1. R. A. Alo and H. L. Shapiro, Paracompact subspaces, Acta Math., 21 (1970).

2. C. E. Aull, A certain class of topological spaces, Prace Matematyczne, 11 (1967), 4953 .

3. — Collectionwise normal subsets, To appear in J. London Math. Soc.

4. - Paracompact subsets, Proceedings of the Second Prague Topological Symposium, (1966), 45-51.

5. _ - Remarks on countable paracompactness, Proc. Japan Acad., 54 (1968), 125126.

6. J. A. Dieudonné, Une généralization des espaces compacts, J. Math. Pures Appl., 23 (1944), 65-76.

7. L. Gillman and M. Jerison, Rings of Continuous Functions, D. Van Nostrand.

8. H. Herrlich, E-kompalkte raume, Math. Zeitschr, 96 (1967), 228-255. 
9. T- Topologische Reflexionen and Coreflexion, Lecture Notes in Mathematics, 78, Springer-Verlag, Berlin, 1968.

10. H. Herrlich and J. Van der Slot, Properties which are closely related to compactness, Indag, Math., 29 (1967), 524-530.

11. E. A. Michael, Another note on paracompact spaces, Proc. Amer. Math. Soc., 8 (1957), 822-823.

12. J. Van der Slot, Some properties related to compactness, Publication of Mathematisch Centrum, Amsterdam, 1968.

13. J. D. Wine, Locally paracompact spaces. To appear.

Received July 14, 1972 and in revised form April 5, 1973.

UNIVERSITY OF WisCONSIN-LA CROSSE 


\section{PACIFIC JOURNAL OF MATHEMATICS}

\section{EDITORS}

RICHARD ARENS (Managing Editor) University of California

Los Angeles, California 90024

R. A. Beaumon'T

University of Washington Seattle, Washington 98105
J. Dugundj1*

Department of Mathematics University of Southern California Los Angeles, California 90007

D. Gilbarg and J. Milgram Stanford University Stanford, California 94305

\section{ASSOCIATE EDITORS}

E. F. BECKENBACH

B. H. NeUMANN

F. WOLF

K. YOSHIDA

\section{SUPPORTING INSTITUTIONS}

UNIVERSITY OF BRITISH COLUMBIA CALIFORNIA INSTITUTE OF TECHNOLOGY UNIVERSITY OF CALIFORNIA MONTANA STATE UNIVERSITY UNIVERSITY OF NEVADA NEW MEXICO STATE UNIVERSITY OREGON STATE UNIVERSITY UNIVERSITY OF OREGON OSAKA UNIVERSITY

\section{UNIVERSITY OF SOUTHERN CALIFORNIA STANFORD UNIVERSITY UNIVERSITY OF TOKYO UNIVERSITY OF UTAH WASHINGTON STATE UNIVERSITY UNIVERSITY OF WASHINGTON AMERICAN MATHEMATICAL SOCIETY NAVAL WEAPONS CENTER}

* C. R. DePrima California Institute of Technology, Pasadena, CA 91109, will replace J. Dugundji until August 1974. 


\section{Pacific Journal of Mathematics}

\section{Vol. 49, No. $1 \quad$ May, 1973}

A. Bigard, Free lattice-ordered modules ...........................

Richard Bolstein and Warren R. Wogen, Subnormal operators in strictly cyclic

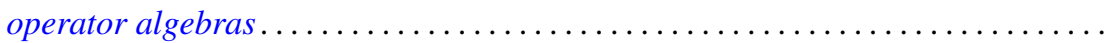

Herbert Busemann and Donald E. Glassco, II, Irreducible sums of simple

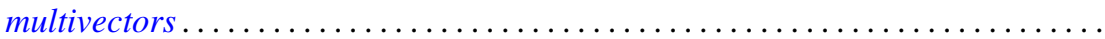

W. Wistar (William) Comfort and Victor Harold Saks, Countably compact groups

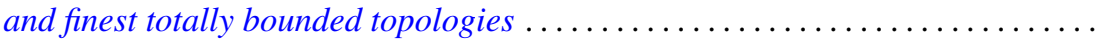

Mary Rodriguez Embry, Maximal invariant subspaces of strictly cyclic operator

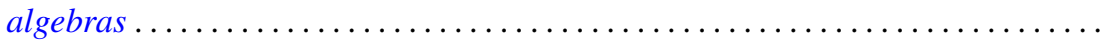

Ralph S. Freese and James Bryant Nation, Congruence lattices of semilattices......

Ervin Fried and George Grätzer, A nonassociative extension of the class of

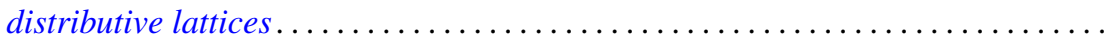

John R. Giles and Donald Otto Koehler, On numerical ranges of elements of locally

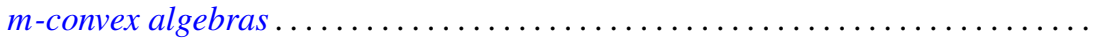

David A. Hill, On dominant and codominant dimension of $\mathrm{QF}-3$ rings ........ John Sollion Hsia and Robert Paul Johnson, Round and Pfister forms over $R(t) \ldots$ I. Martin (Irving) Isaacs, Equally partitioned groups . . . . . . . . . . . . . .

Athanassios G. Kartsatos and Edward Barry Saff, Hyperpolynomial approximation of solutions of nonlinear integro-differential equations.

Shin'ichi Kinoshita, On elementary ideals of $\theta$-curves in the 3-sphere and 2-links in the 4-sphere

Ronald Brian Kirk, Convergence of Baire measures

R. J. Knill, The Seifert and Van Kampen theorem via regular covering spaces ..

Amos A. Kovacs, Homomorphisms of matrix rings into matrix rings ..

Young K. Kwon, HD-minimal but no $H D$-minimal ..........

Makoto Maejima, On the renewal function when some of the mean renewal lifetimes are infinite

Juan José Martínez, Cohomological dimension of discrete modules over profinite groups.

W. K. Nicholson, Semiperfect rings with abelian group of units

Louis Jackson Ratliff, Jr., Three theorems on imbedded prime divisors of principal ideals.

Billy E. Rhoades and Albert Wilansky, Some commutants in $B(c)$ which are almost matrices

John Philip Riley Jr., Cross-sections of decompositions . . .

Keith Duncan Stroyan, A characterization of the Mackey uniformity $m\left(L^{\infty}, L^{1}\right)$ for

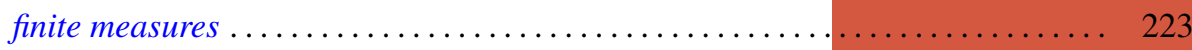

Edward G. Thurber, The Scholz-Brauer problem on addition chains . . . . . . . . . 229

Joze Vrabec, Submanifolds of acyclic 3-manifolds ............

Philip William Walker, Adjoint boundary value problems for compactified singular

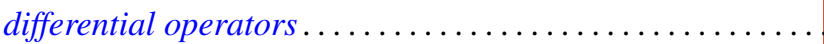

